\title{
SOME MULTIVARIABLE GAUSSIAN HYPERGEOMETRIC EXTENSIONS OF THE PREECE THEOREM
}

\author{
M. I. QURESHI ${ }^{1}$, M. SADIQ KHAN ${ }^{2}$, M. A. PATHAN ${ }^{פ 3}$ and N. U. KHAN ${ }^{3}$
}

(Received 22 July 2002; revised 20 June 2006)

\begin{abstract}
Some generalisations of the Preece theorem involving the product of two Kummer's functions $F_{1}$ are obtained using Dixon's theorem and some well-known identities. Its special cases yield various new transformations and reduction formulae involving Pathan's quadruple hypergeometric function $F_{p}^{(4)}$ and Srivastava's quadruple hypergeometric function $F^{(4)}$ and triple hypergeometric function $F^{(3)}$. Some known results of Preece, Pathan and Bailey are also obtained as special cases.
\end{abstract}

2000 Mathematics subject classification: primary 33C45, 33C55, 33C64, 33C65, 33C70, 33 C99.

Keywords and phrases: Dixon's theorem, Preece's theorem, multiple Gaussian hypergeometric functions.

\section{Introduction}

Let $\left(a_{A}\right)$ denote the sequence of $A$ parameters given by $a_{1}, \ldots, a_{A}$ and $\left[\left(a_{A}\right)\right]_{n}$ denote the product of $A$ Pochhammer symbols defined by

$$
(b)_{n}=\frac{\Gamma(b+n)}{\Gamma(b)}= \begin{cases}1, & \text { if } n=0, \\ b(b+1) \cdots(b+n-1), & \text { if } n=1,2, \ldots\end{cases}
$$

In 1969, Srivastava and Daoust ([11, page 454], see also [12, page $37(21,22)])$

\footnotetext{
'Department of Applied Sciences and Humanities, Faculty of Engineering and Technology, Jamia Millia Islamia, New Delhi-1 10025, India.

${ }^{2}$ Department of Mathematics, Shibli National College, Paharpur, Azamgarh, U.P., India; e-mail: mohdsadiq786@rediffmail.com.

${ }^{3}$ Department of Mathematics, Aligarh Muslim University, Aligarh-202002, India; e-mail: mapathan@gmail.com, nabi_khan@rediffmail.com.

(C) Australian Mathematical Society 2006, Serial-fee code 1446-1811/06
} 
gave the following multivariable hypergeometric function:

$$
\begin{aligned}
& F_{D: E^{(1)} ; \ldots ; E^{(n)}}^{A\left(B^{(1)}\right)}\left[\begin{array}{l}
{\left[\left(a_{A}\right): \theta^{(1)}, \ldots, \theta^{(n)}\right]:\left[\left(b_{B^{(1)}}^{(1)}\right): \Phi^{(1)}\right] ; \ldots ;\left[\left(b_{B^{(n)}}^{(n)}\right): \Phi^{(n)}\right]} \\
{\left[\left(d_{D}\right): \Psi^{(1)}, \ldots, \Psi^{(n)}\right]:\left[\left(e_{E^{(1)}}^{(1)}\right): \delta^{(1)}\right] ; \ldots ;\left[\left(e_{E^{(n)}}^{(n)}\right): \delta^{(n)}\right]}
\end{array} z_{1}, \ldots, z_{n}\right] \\
& =\sum_{m_{1}, \ldots, m_{n}=0}^{\infty} \Xi\left(\mathbf{m}_{1}, \ldots, \mathbf{m}_{n}\right) \frac{z_{1}^{m_{1}}}{\left(m_{1}\right) !} \cdots \frac{z_{n}^{m_{n}}}{\left(m_{n}\right) !},
\end{aligned}
$$

where, for convenience,

$$
\Xi\left(\mathbf{m}_{1}, \ldots, \mathbf{m}_{n}\right)=\frac{\prod_{j=1}^{A}\left(a_{j}\right)_{m_{1} \theta_{j}^{(1)}+\cdots+m_{n} \theta_{j}^{(n)}} \prod_{j=1}^{B^{(1)}}\left(b_{j}^{(1)}\right)_{m_{1} \Phi_{j}^{(1)}} \cdots \prod_{j=1}^{B^{(n)}}\left(b_{j}^{(n)}\right)_{m_{n} \Phi_{j}^{(n)}}}{\prod_{j=1}^{D}\left(d_{j}\right)_{m_{1} \psi_{j}^{(1)}+\cdots+m_{n} \Psi_{j}^{(n)}} \prod_{j=1}^{E^{(1)}}\left(e_{j}^{(1)}\right)_{m_{1} \delta_{j}^{(1)}} \cdots \prod_{j=1}^{E^{(n)}}\left(e_{j}^{(n)}\right)_{m_{n} \delta_{j}^{(n)}}},
$$

the coefficients $\theta_{j}^{(k)}, j=1, \ldots, A ; \Phi_{j}^{(k)}, j=1, \ldots, B^{(k)} ; \Psi_{j}^{(k)}, j=1, \ldots, D ; \delta_{j}^{(k)}$, $j=1, \ldots, E^{(k)}$; for all $k \in\{1, \ldots, n\}$ are zero and real constants (positive, negative) [12, pages 270-272 (Equations 5-9, 19-21)] and $\left(b_{B^{(k)}}^{(k)}\right)$ abbreviates the array of $B^{(k)}$ parameters $b_{j}^{(k)}, j=1, \ldots, B^{(k)}$; for all $k \in\{1, \ldots, n\}$, with similar interpretations for others.

Preece [7, page 378 (11)] gave the following formula for the product of two hypergeometric functions:

$$
\begin{aligned}
{ }_{1} F_{1}\left[\begin{array}{ll}
c & y \\
f & y
\end{array}\right]{ }_{1} F_{1}\left[\begin{array}{cc}
1-f+c & -y \\
2-f & -f
\end{array}\right] \\
\quad={ }_{2} F_{3}\left[\begin{array}{cc}
1 / 2+f / 2-c, 1 / 2-f / 2+c & y^{2} / 4 \\
f / 2+1 / 2,3 / 2-f / 2,1 / 2 &
\end{array}\right] \\
\quad+\frac{(f-1)(f-2 c) y}{f(2-f)}{ }_{2} F_{3}\left[\begin{array}{cc}
1-c+f / 2,1+c-f / 2 & y^{2} / 4 \\
2-f / 2,1+f / 2,3 / 2 &
\end{array}\right] .
\end{aligned}
$$

The present paper is devoted to the investigation of general multiple series identities which extend and generalise the theorems of Bailey [1, page 239 (4.6)], Pathan [5, page 1115 (3.2)] and Preece [7, page 378 (11)]. These theorems given in Section 2 will be seen to be extremely useful, in that most properties of hypergeometric series carry over naturally and simply for these identities and provide connections with various classes of well-known hypergeometric functions and even new representations for special cases of these functions. Some applications of these theorems are given in

Section 3. Clearly, the same procedure could have been utilised to extend many more results on hypergeometric functions. But, instead, we deduce some special cases in Section 4.

\section{Multivariable analogues of the Preece theorem}

Motivated by the works of Bailey, Pathan and Preece, we shall establish the following theorems for multiple series, which are more generalised than the multiple 
Gaussian hypergeometric functions $F^{(3)}, F^{(4)}$ and $F_{P}^{(4)}$.

THEOREMS. Let $S(i, j, k, p)$ be the generalised coefficients of arbitrary complex numbers, where $x, y, z$ are complex variables and $c, f$ are arbitrary independent complex parameters (where $2 f \neq 0,-1, \pm 2, \pm 3, \ldots$ ) and any values of numerator and denominator parameters and variables $x, y, z$ leading to results which do not make sense are tacitly excluded, then

$$
\begin{aligned}
\sum_{i, j, k, p=0}^{\infty} & S(i+k, j, p) \frac{(-1)^{k}(c-2 f+1)_{i}(c)_{k} x^{j} y^{i+k} z^{p}}{(2-2 f)_{i}(2 f)_{k} i ! j ! k ! p !} \\
= & \sum_{t=0}^{1} \frac{y^{t}}{t !} \Omega(t) \sum_{i, j, p=0}^{\infty} S(2 i+t, j, p) \Lambda(t, i) \frac{\left(y^{2} / 4\right)^{i} x^{j} z^{p}}{j ! p !} \\
= & \sum_{t, u=0}^{1} \frac{y^{t} x^{u}}{t ! u !} \Omega(t) \sum_{i, j, p=0}^{\infty} S(2 i+t, 2 j+u, p) \Lambda(t, i) \\
& \times \frac{\left(y^{2} / 4\right)^{i}\left(x^{2} / 4\right)^{j} z^{p}}{((1+u) / 2)_{j}(1+u / 2)_{j} p !} \\
= & \sum_{t, u, w=0}^{1} \frac{y^{t} x^{u} z^{w}}{t ! u ! w !} \Omega(t) \sum_{i, j, p=0}^{\infty} S(2 i+t, 2 j+u, 2 p+w) \Lambda(t, i) \\
& \times \frac{\left(y^{2} / 4\right)^{i}\left(x^{2} / 4\right)^{j}\left(z^{2} / 4\right)^{p}}{((1+u) / 2)_{j}(1+u / 2)_{j}((1+w) / 2)_{p}(1+w / 2)_{p}},
\end{aligned}
$$

where, for convenience,

$$
\begin{aligned}
\Omega(t) & =\frac{\Gamma(2 f) \Gamma\left(\frac{1+2 f-t}{2}\right) \Gamma\left(\frac{1+2 f-2 c+t}{2}\right) \Gamma(2 f-c-t)(c-2 f+1)_{t}}{\Gamma(2 f-c) \Gamma\left(\frac{1+2 f+t}{2}\right) \Gamma\left(\frac{1+2 f-2 c-t}{2}\right) \Gamma(2 f-t)(2-2 f)_{t}}, \\
\Lambda(t, i) & =\frac{\left(\frac{1+2 f-2 c+t}{2}\right)_{i}\left(\frac{1-2 f+2 c+t}{2}\right)_{i}}{\left(\frac{3-2 f+t}{2}\right)_{i}\left(\frac{1+2 f+t}{2}\right)_{i}\left(\frac{1+t}{2}\right)_{i}\left(\frac{2+t}{2}\right)_{i}},
\end{aligned}
$$

provided that each multiple series involved converges absolutely.

ProOF OF THEOREMS (2.1)-(2.3). Let $L$ denote the left-hand side of (2.1). Then using the series identity $[8$, page 56 , Lemma $10(1)]$ defined as

$$
\sum_{i, j, k, p=0}^{\infty} A(i, j, k, p)=\sum_{i, j, p=0}^{\infty} \sum_{k=0}^{i} A(i-k, j, k, p),
$$

we may write

$$
L=\sum_{i, j, p=0}^{\infty} S(i, j, p) \frac{(c-2 f+1)_{i} y^{i} x^{j} z^{p}}{(2-2 f)_{i} i ! j ! p !}{ }_{3} F_{2}\left[\begin{array}{c}
2 f-1-i,-i, c \\
2 f, 2 f-c-i
\end{array}\right] .
$$


Using Dixon's theorem [8, page 92, Theorem 33] in (2.5), we may then write

$$
\begin{aligned}
L= & \sum_{i, j, p=0}^{\infty} S(i, j, p) \frac{(c-2 f+1)_{i} y^{i} x^{j} z^{p}}{(2-2 f)_{i} i ! j ! p !} \\
& \times \frac{\Gamma(2 f) \Gamma\left(\frac{1+2 f-i}{2}\right) \Gamma\left(\frac{1+2 f-2 c+i}{2}\right) \Gamma(2 f-c-i)}{\Gamma\left(\frac{1+2 f-2 c-i}{2}\right) \Gamma\left(\frac{1+2 f+i}{2}\right) \Gamma(2 f-i) \Gamma(2 f-c)} .
\end{aligned}
$$

Now applying the following identities [13, page 214 (8), page 217 (12)]:

$$
\begin{aligned}
\sum_{i=0}^{\infty} A(i) & =\sum_{i=0}^{1} \sum_{i=0}^{\infty} A(2 i+t), \\
\sum_{i, j=0}^{\infty} B(i, j) & =\sum_{i, u=0}^{1} \sum_{i, j=0}^{\infty} B(2 i+t, 2 j+u), \\
\sum_{i, j, p=0}^{\infty} C(i, j, p) & =\sum_{t, u, w=0}^{1} \sum_{i, j, p=0}^{\infty} C(2 i+t, 2 j+u, 2 p+w)
\end{aligned}
$$

in (2.6), then using the series rearrangement technique, we get the right-hand sides of (2.1)-(2.3) respectively.

\section{Applications of Theorems (2.1) - (2.3)}

3.1. The Preece Theorem associated with Srivastava's function $F^{(3)}$. In Theorems (2.1) and (2.2), setting

$$
S(i, j, k)=\frac{\left[\left(a_{A}\right)\right]_{i+j+k}\left[\left(d_{D}\right)\right]_{i+k}\left[\left(g_{G}\right)\right]_{j}}{\left[\left(b_{B}\right)\right]_{i+j+k}\left[\left(e_{E}\right)\right]_{i+k}\left[\left(h_{H}\right)\right]_{j}}
$$

and $z=0$ and using the series rearrangement technique, we get

$$
\begin{aligned}
& F^{(3)}\left[\begin{array}{rr}
\left(a_{A}\right)::-;\left(d_{D}\right) ;-:\left(g_{G}\right) ; c-2 f+1 ; c ; \\
\left(b_{B}\right)::-;\left(e_{E}\right) ;-:\left(h_{H}\right) ; & 2-2 f ; 2 f ;
\end{array} \quad x, y,-y\right] \\
& =\sum_{t=0}^{1} \frac{\left[\left(a_{A}\right)\right]_{t}\left[\left(d_{D}\right)\right]_{t} y^{t}}{\left[\left(b_{B}\right)\right]_{t}\left[\left(e_{E}\right)\right]_{t} t !} \Omega(t) \\
& \times F_{B: 2 E+3: H}^{A: 2 D+2: G}\left[\begin{array}{l}
{\left[\left(a_{A}\right)+t: 2,1\right]:\left[\Delta\left(2 ;\left(d_{D}+t\right): 1\right],\left[\frac{1+t}{2}+f-c: 1\right],\right.} \\
{\left[\left(b_{B}\right)+t: 2,1\right]:\left[\Delta\left(2 ;\left(e_{E}+t\right): 1\right],\left[\frac{3+t}{2}-f: 1\right]\right.}
\end{array},\right. \\
& \begin{array}{l}
\left.\left[\frac{1+t}{2}-f+c: 1\right] ;\left[\left(g_{G}\right): 1\right] ; y^{2} / 4^{1+E-D}, x\right] \\
-c: 1],\left[\frac{1+t}{2}: 1\right] ;\left[\left(h_{H}\right): 1\right] ;
\end{array}
\end{aligned}
$$




$$
\begin{aligned}
& =\sum_{t, u=0}^{1} \frac{\left[\left(a_{A}\right)\right]_{t+u}\left[\left(d_{D}\right)\right]_{t}\left[\left(g_{G}\right)\right]_{u} y^{t} x^{u}}{\left[\left(b_{B}\right)\right]_{t+u}\left[\left(e_{E}\right)\right]_{t}\left[\left(h_{H}\right)\right]_{u} t ! u !} \Omega(t) \\
& \times F_{2 B: 2 E+3 ; 2 H+1}^{2 A: 2 D+2,2 C}\left[\begin{array}{l}
\Delta\left[2 ;\left(a_{A}\right)+t+u\right] ; \frac{1+t}{2}+f-c, \frac{1+t}{2}-f+c, \\
\Delta\left[2 ;\left(b_{B}\right)+t+u\right] ; \frac{3}{2}-f+\frac{t}{2}, \frac{1}{2}+f+\frac{t}{2}, \Delta^{*}(2 ; 1+t),
\end{array}\right. \\
& \Delta\left[2 ;\left(d_{D}\right)+t\right] ; \Delta\left[2 ;\left(g_{G}\right)+u\right] ; \\
& \left.\Delta\left[2 ;\left(e_{E}\right)+t\right] ; \Delta^{*}(2 ; 1+u), \Delta\left[2 ;\left(h_{H}\right)+u\right] ; \frac{4^{1+B+E}}{4^{1+B+H}}\right] \text {, }
\end{aligned}
$$

provided that the denominator parameters are neither zero nor negative integers and for convenience, the symbol $\Delta(m ; b)$ abbreviates the array of $m$ parameters given by

$$
\frac{b}{m}, \frac{(b+1)}{m}, \frac{(b+2)}{m}, \ldots, \frac{(b+m-1)}{m},
$$

where $m=1,2,3, \ldots$

The asterisk in $\Delta^{*}(N ; j+1)$ represents the fact that the (denominator) parameter $N / N$ is always omitted for $0 \leq j \leq(N-1)$, so that the set $\Delta^{*}(N ; j+1)$ obviously contains only $N-1$ parameters [13, page 214].

The notation $\Delta\left[N ;\left(b_{B}\right)\right]$ denotes the array of $B N$ parameters [13, page $47(8)$, pages 193-194] given by $\Delta\left(N ; b_{1}\right), \ldots, \Delta\left(N ; b_{B}\right)$ and $\Omega(t)$ is given by $(2.4)$.

3.2. The Preece theorem associated with Srivastava's function $F^{(4)}$. In Theorem (2.3), setting

$$
S(i, j, k, p)=\frac{\left[\left(a_{A}\right)\right]_{i+j+k+p}\left[\left(m_{M}\right)\right]_{j+p}\left[\left(d_{D}\right)\right]_{i+k}\left[\left(g_{G}\right)\right]_{j}\left[\left(q_{Q}\right)\right]_{p}}{\left[\left(b_{B}\right)\right]_{i+j+k+p}\left[\left(n_{N}\right)\right]_{j+p}\left[\left(e_{E}\right)\right]_{i+k}\left[\left(h_{H}\right)\right]_{j}\left[\left(r_{R}\right)\right]_{p}}
$$

we get

$$
\begin{aligned}
& F^{(4)}\left[\begin{array}{l}
\left(a_{A}\right):: c-2 f+1 ;\left(d_{D}\right) ;\left(g_{G}\right) ;\left(m_{M}\right): c ;\left(d_{D}\right) ;\left(q_{Q}\right) ;\left(m_{M}\right) ; y, x,-y, z \\
\left(b_{B}\right):: 2-2 f \quad ;\left(e_{E}\right) ;\left(h_{H}\right) ;\left(n_{N}\right): 2 f ;\left(e_{E}\right) ;\left(r_{R}\right) ;\left(n_{N}\right) ;
\end{array}\right] \\
& =\sum_{t, u, w=0}^{1} \frac{\left[\left(a_{A}\right)\right]_{t+u+w}\left[\left(m_{M}\right)\right]_{u+w}\left[\left(d_{D}\right)\right]_{t}\left[\left(g_{G}\right)\right]_{u}\left[\left(q_{Q}\right)\right]_{w} y^{t} x^{u} z^{w}}{\left[\left(b_{B}\right)\right]_{t+u+w}\left[\left(n_{N}\right)\right]_{u+w}\left[\left(e_{E}\right)\right]_{t}\left[\left(h_{H}\right)\right]_{u}\left[\left(r_{R}\right)\right]_{w} t ! u ! w !} \Omega(t) \\
& \times F^{(3)}\left[\begin{array}{l}
\Delta\left[2 ;\left(a_{A}\right)+t+u+w\right]::-; \Delta\left[2 ;\left(m_{M}\right)+u+w\right] ;-: \\
\Delta\left[2 ;\left(b_{B}\right)+t+u+w\right]::-; \Delta\left[2 ;\left(n_{N}\right)+u+w\right] ;-:
\end{array}\right. \\
& \frac{1+t}{2}+f-c, \frac{1+t}{2}-f+c, \Delta\left[2 ;\left(d_{D}\right)+t\right] \\
& \frac{3+t}{2}-f, \frac{1+t}{2}+f, \Delta^{*}(2 ; 1+t), \Delta\left[2 ;\left(e_{E}\right)+t\right] \text {; } \\
& \Delta\left[2 ;\left(g_{G}\right)+u\right] ; \Delta\left[2 ;\left(q_{Q}\right)+w\right] ; \\
& \Delta^{*}(2 ; 1+u), \Delta\left[2 ;\left(h_{H}\right)+u\right] ; \Delta^{*}(2 ; 1+w), \Delta\left[2 ;\left(r_{R}\right)+w\right] ; \\
& \left.\frac{4^{A+D} y^{2}}{4^{1+B+E}}, \frac{4^{A+G+M} x^{2}}{4^{1+B+H+N}}, \frac{4^{A+M+Q} z^{2}}{4^{1+B+N+R}}\right] \text {. }
\end{aligned}
$$


3.3. The Preece theorem associated with Pathan's function $F_{P}^{(4)}$. In Theorem (2.3), setting

$$
S(i, j, k, p)=\frac{\left[\left(a_{A}\right)\right]_{j+p+i+k}\left[\left(g_{G}\right)\right]_{i+k+j}\left[\left(d_{D}\right)\right]_{p+i+k}\left[\left(m_{M}\right)\right]_{j}\left[\left(q_{Q}\right)\right]_{p}}{\left[\left(b_{B}\right)\right]_{j+p+i+k}\left[\left(h_{H}\right)\right]_{i+k+j}\left[\left(e_{E}\right)\right]_{p+i+k}\left[\left(n_{N}\right)\right]_{j}\left[\left(r_{R}\right)\right]_{p}}
$$

we get

$$
\begin{gathered}
F_{P}^{(4)}\left[\begin{array}{c}
\left.\left(a_{A}\right)::-;\left(d_{D}\right) ;\left(g_{G}\right) ;-:\left(m_{M}\right) ;\left(q_{Q}\right) ; c-2 f+1 ; c ; x, z, y,-y\right] \\
\left(b_{B}\right)::-;\left(e_{E}\right) ;\left(h_{H}\right) ;-:\left(n_{N}\right) ;\left(r_{R}\right) ; 2-2 f \quad ; 2 f ;
\end{array}\right] \\
=\sum_{t, u, w=0}^{1} \frac{\left[\left(a_{A}\right)\right]_{t+u+w}\left[\left(d_{D}\right)\right]_{t+w}\left[\left(g_{G}\right)\right]_{t+u}\left[\left(m_{M}\right)\right]_{u}\left[\left(q_{Q}\right)\right]_{w} y^{t} x^{u} z^{w}}{\left[\left(b_{B}\right)\right]_{t+u+w}\left[\left(e_{E}\right)\right]_{t+w}\left[\left(h_{H}\right)\right]_{t+u}\left[\left(n_{N}\right)\right]_{u}\left[\left(r_{R}\right)\right]_{w} t ! u ! w !} \Omega(t) \\
\times F^{(3)}\left[\begin{array}{l}
\Delta\left[2 ;\left(a_{A}\right)+t+u+w\right]::-; \Delta\left[2 ;\left(d_{D}\right)+t+w\right] ; \Delta\left[2 ;\left(g_{G}\right)+t+u\right]: \\
\Delta\left[2 ;\left(b_{B}\right)+t+u+w\right]::-\Delta\left[2 ;\left(e_{E}\right)+t+w\right] ; \Delta\left[2 ;\left(h_{H}\right)+t+u\right]: \\
\Delta\left[2 ;\left(m_{M}\right)+u\right] \quad ; \Delta\left[2 ;\left(q_{Q}\right)+w\right] ; \\
\Delta^{*}(2 ; 1+u), \Delta\left[2 ;\left(n_{N}\right)+u\right] ; \Delta^{*}(2 ; 1+w), \Delta\left[2 ;\left(r_{R}\right)+w\right] ; \\
\frac{1+t}{2}+f-c, \frac{1+t}{2}-f+c \quad ; \\
\frac{3+t}{2}-f, \frac{1+t}{2}+f, \Delta^{*}(2 ; 1+t) ; \\
\left.\frac{4^{A+G+M} x^{2}}{4^{1+B+H+N}}, \frac{4^{A+D+Q} z^{2}}{4^{1+B+E+R}}, \frac{4^{A+G+D} y^{2}}{4^{1+B+H+E}}\right] .
\end{array}\right.
\end{gathered}
$$

Here the double hypergeometric function $F_{B: E ; H}^{A ; D ; G}$, the triple hypergeometric function $F^{(3)}$, and the quadruple hypergeometric functions $F^{(4)}$ and $F_{p}^{(4)}$ were given by Kampé de Fériet, see [12, page 27(28)], Srivastava [10, page 428], [9, pages 35-36(1.2)] and Pathan [6, page 172(1.2)], respectively.

\section{Special cases}

(I) Setting $A=M=H=R=1, a_{1}=a, m_{1}=m, h_{1}=h, r_{1}=r$ and $B=D=E=G=N=Q=0$ in (3.1), we get

$$
\begin{aligned}
& K_{10}[a, a, a, a ; m, m, c-2 f+1, c ; h, r, 2-2 f, 2 f ; x, z, y,-y] \\
& =\sum_{t . u, w=0}^{1} \frac{(a)_{t+u+w}(m)_{u+w} y^{t} x^{u} z^{w}}{(h)_{u}(r)_{w} t ! u ! w !} \Omega(t) \\
& \times F^{(3)}\left[\begin{array}{l}
\Delta(2 ; a+t+u+w):-; \Delta(2 ; m+u+w) ;-: \\
\quad:-;-: \\
\quad 3 / 2-f+t / 2,1 / 2+f+t / 2 \quad ; \Delta^{*}(2 ; 1+t) ; \Delta^{*}(2 ; 1+u),
\end{array}\right.
\end{aligned}
$$




$$
\left.\Delta(2 ; h+u) ; \Delta^{*}(2 ; 1+w), \Delta(2 ; r+w) ; y^{2}, x^{2}, z^{2}\right]
$$

(II) Setting $A=N=Q=G=1, a_{1}=a, n_{1}=n, g_{1}=g, q_{1}=s$ and $B=D=E=M=H=R=0$ in (3.1), we get

$$
\begin{aligned}
& K_{13}[a, a, a, a ; g, s, c-2 f+1, c ; n, n, 2-2 f, 2 f ; x, z, y,-y] \\
& =\sum_{t, u, w=0}^{1} \frac{(a)_{t+u+w}(g)_{u}(s)_{w} y^{t} x^{u} z^{w}}{(n)_{u+w} t ! u ! w !} \Omega(t) \\
& \times F^{(3)}\left[\begin{array}{rl}
\Delta(2 ; a+t+u+w) & ::-;-; \\
::-;-; \Delta(2 ; n+u+w) ; \Delta^{*}(2 ; 1+u) ;
\end{array}\right. \\
& \begin{array}{ll}
\frac{1}{2}+f-c+\frac{t}{2}, \frac{1}{2}-f+c+\frac{t}{2} & \left.; \Delta(2 ; s+w) ; x^{2}, y^{2}, z^{2}\right] . \\
\frac{3}{2}-f+\frac{t}{2}, \frac{1}{2}+f+\frac{t}{2}, \Delta^{*}(2 ; 1+t) ; \Delta^{*}(2 ; 1+w) ;
\end{array}
\end{aligned}
$$

(III) Setting $A=M=N=Q=R=1, a_{1}=a, m_{1}=m, n_{1}=n, q_{1}=q, r_{1}=r$ and $B=D=E=G=H=0$ in (3.2), we get

$$
\begin{aligned}
F_{A}^{(4)}[a, c, q, & m, c-2 f+1 ; 2 f, r, n, 2-2 f ;-y, z, x, y] \\
= & \sum_{t, u, w=0}^{1} \frac{(a)_{t+u+w}(m)_{u}(q)_{w} y^{t} x^{u} z^{w}}{(n)_{u}(r)_{w} t ! u ! w !} \Omega(t) \\
\times & F^{(3)}\left[\begin{array}{l}
\Delta(2 ; a+t+u+w)::-;-;-: \Delta(2 ; m+u) \\
\frac{\Delta\left(:-;-: \Delta^{*}(2 ; 1+u), \Delta(2 ; n+u) ;\right.}{\Delta(2 ; q+w)} ; \\
\Delta^{*}(2 ; 1+w), \Delta(2 ; r+w) ; \\
\frac{1+t}{2}+f-c, \frac{1+t}{2}-f+c \\
\left.\frac{3+t}{2}-f, \frac{1+t}{2}+f, \Delta^{*}(2 ; 1+t) ; x^{2}, z^{2}, y^{2}\right] .
\end{array}\right.
\end{aligned}
$$

(IV) In (2.1), setting $S(i, j, k)=(a)_{2 j+i+k} /(b)_{j}$ and $z=0$, we get

$$
\begin{aligned}
& X_{8}[a, c-2 f+1, c ; b, 2-2 f, 2 f ; x, y,-y] \\
& =\sum_{t=0}^{1} \frac{y^{t}(a)_{t}}{t !} \Omega(t) \\
& \times F_{0: 3 ; 1}^{2: 2 ; 0}\left[\begin{array}{r}
\Delta(2 ; a+t): \frac{1+t}{2}+f-c, \frac{1+t}{2}-f+c \quad ;-; y^{2}, 4 x \\
: \frac{3+t}{2}-f, \frac{1+t}{2}+f, \Delta^{*}(2 ; 1+t) ; b ;
\end{array}\right] .
\end{aligned}
$$

(V) In (2.1), setting $S(j, p, i, k)=(a)_{2 j+2 p+i+k} /(b)_{j}(d)_{j}$, we get

$$
{ }^{(2)} H_{4}^{(4)}[a, c-2 f+1, c ; b, d, 2-2 f, 2 f ; x, z, y,-y]
$$




$$
\begin{aligned}
& =\sum_{t=0}^{1} \frac{y^{t}(a)_{t}}{t !} \Omega(t) \\
& \times F^{(3)}\left[\begin{array}{r}
\Delta(2 ; a+t)::-;-;-:-;-; \\
::-;-;-: b ; d ;
\end{array}\right. \\
& \begin{array}{l}
\frac{1+t}{2}+f-c, \frac{1+t}{2}-f+c \\
\left.\frac{3+t}{2}-f, \frac{1+t}{2}+f, \Delta^{*}(2 ; 1+t) ; 4 x, 4 z, y^{2}\right] .
\end{array}
\end{aligned}
$$

Here $K_{10}, K_{13}, X_{8},{ }^{(2)} H_{4}^{(4)}$ and $F_{A}^{(4)}$ are the multiple Gaussian hypergeometric functions of Exton [2, page 79 (3.3.10, 3.3.13)], [3, page $113(1.8)$ ], [2, page 97 (3.5.2)] and Lauricella [4, page 150], respectively.

Acknowledgements The authors are thankful to the referee for his valuable comments and suggestions leading to a better presentation of the paper.

\section{References}

[1] W. N. Bailey, "On the sum of a terminating ${ }_{3} F_{2}(1)$ ", Quart J. Math. (Oxford) Series (2) 4 (1953) 237-240.

[2] H. Exton, Multiple hypergeometric functions and applications (Halsted Press (Ellis Horwood, Chichester, UK), John Wiley and Sons, New York, Chichester, Brisbane and Toronto, 1976).

[3] H. Exton, "Hypergeometric functions of three variables", J. Indian Acad. Math. 4 (1982) 113-119.

[4] G. Lauricella, "Sulle funzioni ipergeometriche a piu variabili", Rend. Circ. Mat. Palermo 7 (1893) $111-158$.

[5] M. A. Pathan, "On some transformations of triple hypergeometric series $F^{(3)}$-III", Indian J. Pure Appl. Math. 9 (1978) 1113-1117.

[6] M. A. Pathan, "On a transformation of a general hypergeometric series of four variables", Nederl. Akad. Wetensch. Proc. Ser. A, 82 = Indag. Math. 41 (1979) 171-175.

[7] C. T. Preece, "The product of two generalized hypergeometric functions", Proc. London Math. Soc. (2) 22 (1924) 370-380.

[8] E. D. Rainville, Special functions (Macmillan, New York, 1960; Reprinted by Chelsea, Bronx, New York, 1971).

[9] H. M. Srivastava, “A formal extension of certain generating functions-II", Glasnik Mat. Ser. III 26 (1971) 35-44.

[10] H. M. Srivastava, "Generalized Neumann expansions involving hypergeometric functions", Proc. Cambridge Philos. Soc. 63 (1976) 445-429.

[11] H. M. Srivastava and M. C. Daoust, "Certain generalized Neumann expansions associated with Kampé de Fériet function”, Nederl. Akad. Wetensch. Proc. Ser. A, $72=$ Indag. Math. 31 (1969) $449-457$.

[12] H. M. Srivastava and P. W. Karlsson, Multiple Gaussian Hypergeometric Series (Halsted Press (Ellis Horwood, Chichester, UK), John Wiley and Sons, New York, Chichester, Brisbane and Toronto, 1985).

[13] H. M. Srivastava and H. L. Manocha, A Treatise on Generating Functions (Halsted Press (Ellis Horwood, Chichester, UK), John Wiley and Sons, New York, Chichester, Brisbane and Toronto, 1984). 